of the vibrations of the magnetic ion alone, $\Delta_{0}$, at $T=0$ is $1.2 \%$ of the observed zero-field splitting. Similarly, we find $\Delta_{0} / D=0.027$ at room temperatures. For ${ }^{54} \mathrm{Fe}^{3+}, 56 \mathrm{Fe}^{3+}$, and ${ }^{57} \mathrm{Fe}^{3+}$ in calcite, we use $T_{\mathrm{D}}=470^{\circ} \mathrm{K}[8]$, and obtain $\Delta_{0} / D=0.007$ at $T=0^{\circ} \mathrm{K}$ and 0.03 at $300^{\circ} \mathrm{K}$. We fail to account for the temperature independence of $\delta \Delta_{0}$ from $4.2^{\circ} \mathrm{K}$ as found experimentally [ 1 ]. Instead, our calculations show that $\delta U$ decreases by a factor of 2 at $T=112^{\circ} \mathrm{K}^{*}$. For ${ }^{155} \mathrm{Gd}^{3+}$ and $157 \mathrm{Gd}^{3+}$ in $\mathrm{ThO}_{2}$, as before [4], we assume that the lattice vibrations pertinent to our problem may be represented by the vibrations of the Th ions alone with [9] $T_{\mathrm{D}}=270^{\circ} \mathrm{K}$. In this case, we get $\Delta_{0} / D=0.006$ at $T=0^{\circ} \mathrm{K}$ and 0.033 at $T=$ $=300^{\circ} \mathrm{K}$. We also find that the isotope shift approximately decreases by a factor of 2 at $200^{\circ} \mathrm{K}$. This result agrees with Marshall's experimental data [3]. Up to now, we have only considered the motion of the magnetic ions alone. If we take into account the motion of the neighboring ions, we might expect the phonon-induced splitting at $300^{\circ} \mathrm{K}$ to be more than $10 \%$ of the observed zerofield splitting. However, in many cases $[1-3,10]$,

* This could be due to that the elastic constant around the impurity ion is smaller than that of the perfect crystal. the measured zero-field splittings decreases with temperature, and hence we might conclude that the orbit-lattice interaction is by no means the dominant mechanism responsible for the zerofield splitting.

We would like to thank Mrs. Margery Ratner for helping with the calculations.

\section{References}

1. S. A. Marshall, J.A. Hodges and R. A. Serway. Phys. Rev. 133 (1964) A1427.

2. S. A. Marshall. J.A. Hodges and R. A. Serway, Phys. Rev. 136 (1964) A1024.

3. S. A. Marshall, Phys. Rev. 159 (1967) 191.

4. C.-Y.Huang, Phys. Rev. 168 (1968) 334.

5. A. A. Maradudin and P.A.Flinn, Phys. Rev, 126 (1962) 2059; W. M. Visscher. Phys. Rev. 129 (1963) 28.

6. P.G.Dawber and R.J.Elliott, Proc.Roy. Soc. 273 (1963) 222 .

7. C.Susse, J.Recherches 12 (1961) 21.

8. L. Peselnick and R. A. Robie, J.Appl. Phys. 33 (1962) 2889 .

9. B. T.M. Willis, Proc. Roy. Soc. (London) A274 (1963) 134.

10. See also Fahd George Wakim, Ph. D.thesis. University of Texas, 1964.

\title{
STRESS DEPENDENT FREQUENCY SHIFT OF THE RESONANT BANDMODE IN $\mathrm{NaCl}: \mathrm{Cu}^{+}$
}

G. BUSSE, W. PRETTL and L. GENZEL

Physikalisches Institut der Universität Freiburg, i.Br. , Germany

Received 4 June 1968

\begin{abstract}
The symmetry of the resonant bandmode and the anharmonic coupling coefficients in $\mathrm{NaCl}: \mathrm{Cu}^{+}$have been
\end{abstract} determined from the stress dependent shift of the resonance frequency.

Uniaxial stress induces large frequency shifts of resonant bandmodes [1] and allows determination of the symmetry of the impurity mode and of the anharmonic coupling coefficients.

We investigated the stress behaviour of the optically active resonant bandmode in $\mathrm{NaCl}: \mathrm{Cu}^{+}$ [2] at $\nu_{\mathbf{R}}=23.6 \mathrm{~cm}^{-1}$.

The transmission measurements were per- formed with a periodically working far-infrared interferometric modulator [3], the radiation being polarized with a 100 lines per $\mathrm{cm}$ wire grid and detected by a Ge-bolometer *. The resolution was $0.4 \mathrm{~cm} \mathrm{~cm}^{-1}$.

The concentration of $\mathrm{Cu}^{+}$- natural mixture of

* Texas Instruments Inc. 
isotopes - was determined to be about $10^{17} \mathrm{Cu}^{+} / \mathrm{cm}^{3}$.

A cryostat with exchange gas chamber was constructed to maintain $4.3^{\circ} \mathrm{K}$ during all measurements and to apply uniaxial stress up to about $3 \mathrm{~kg} / \mathrm{mm}^{2}$ to crystals of $3 \mathrm{~cm}^{2}$ area and thickness of $6 \mathrm{~mm}$ to $12 \mathrm{~mm}$. The stress was produced by bellows the interior of which was coupled to the cryostate vacuum, while the exterior gas pressure upon the bellows was adjustable up to $1 \mathrm{~kg} / \mathrm{cm}^{2}$.

The stress was applied along [100] and [110] crystallographic orientations. The frequency of the resonant bandmode was measured for different directions of beam incidence and polarisation of the radiation. The results are shown in fig. 1; the experimental conditions and symmetries are explained in table 1 .

The shift of the resonance frequency is linear in stress within the accuracy of measurement. The crystal deformation was controlled and proved to be proportional to stress and reversible up to about $P=2 \mathrm{~kg} / \mathrm{mm}^{2}$ where plastic deformation began.

The splitting of the resonant mode corresponds to the following symmetry properties: The normal coordinates of the mode transform like $\Gamma_{4}{ }^{-}$representations of the $\mathrm{O}_{\mathrm{h}}$-group. Uniaxial stress along [100] lowers symmetry to $D_{4 h}$, along [110] to $\mathrm{D}_{2} \mathrm{~h}$. The threefold degenerated $\Gamma_{4}{ }^{-}$state is compatible with $\Gamma_{2}{ }^{-}$and $\Gamma_{5}{ }^{-}$(twofold) of $D_{4 h}$ and

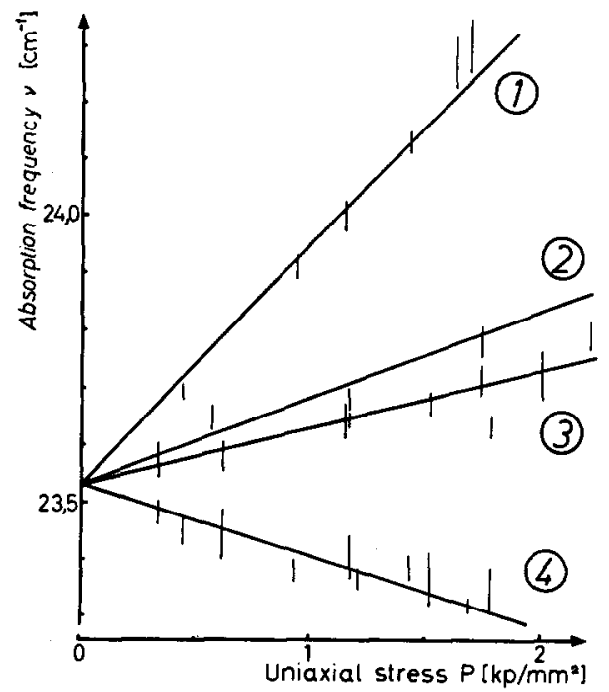

Fig. 1. Frequency $\nu$ of the resonant bandmode as a function of stress $P$. The numbers correspond to the indices in table 1 .
Table 1

Experimental conditions and corresponding symmetries.

\begin{tabular}{|c|c|c|c|c|c|}
\hline Index & Stress & Beam & $E$-vector & Symm. & Repr. \\
\hline \multirow[t]{2}{*}{1} & & & {$[100]$} & & $\Gamma_{2}^{-}$ \\
\hline & {$[100]$} & {$[001]$} & & $\mathrm{D}_{4 \mathrm{~h}}$ & \\
\hline 4 & & & [010] & & $\Gamma \overline{5}$ \\
\hline \multirow[t]{2}{*}{3} & & & {$[110]$} & & $\Gamma_{4}^{-}$ \\
\hline & {$[110]$} & [1̄10] & & $\mathrm{D}_{2 \mathrm{~h}}$ & \\
\hline 4 & & & [001] & & $\Gamma_{2}^{-}$ \\
\hline \multirow[t]{2}{*}{3} & & & {$[110]$} & & $\Gamma_{4}^{-}$ \\
\hline & [110] & {$[001]$} & & $\mathrm{D}_{2 \mathrm{~h}}$ & \\
\hline 2 & & & {$[\overline{1} 10]$} & & $\Gamma_{3}^{-}$ \\
\hline
\end{tabular}

with $\Gamma_{2}^{-}, \Gamma_{3}^{-}$, and $\Gamma_{4}^{-}$of $\mathrm{D}_{2 h}[4]$ which are the irreducible representations of the normal coordinates in the stress deformed cyrstal.

The anharmonic coupling coefficients $[1,5]$ of the resonant bandmode to static distortions or low frequency lattice, modes belonging to $\Gamma_{1}^{+}, \Gamma_{3}^{+}$and $\Gamma_{5}^{+}$representations, can be calculated from the slope of the frequency versus stress curves. We find

$\Gamma_{1}^{+}: A\left(S_{11}+S_{12}\right)=0.043 \mp 0.017 \mathrm{~cm}^{-1} \mathrm{~kg}^{-1} \mathrm{~mm}^{2}$

$\Gamma_{3}^{+}: B\left(S_{11}-S_{12}\right)=0.090 \mp 0.007 \mathrm{~cm}^{-1} \mathrm{~kg}^{-1} \mathrm{~mm}^{2}$

$\Gamma_{5}^{+}: C S_{44} \quad=-0.062 \mp 0.039 \mathrm{~cm}^{-1} \mathrm{~kg}^{-1} \mathrm{~mm}^{2}$

with $S_{11}, S_{12}$ and $S_{44}$ being the local compliances at the impurity site which can be obtained by a correction [6] from the pure $\mathrm{NaCl}$ low temperature compliances [7].

Thereupon we get $A=230 \mathrm{~cm}^{-1} \pm 40 \%, B=$ $=170 \mathrm{~cm}^{-1} \pm 8 \%, C=-80 \mathrm{~cm}^{-1} \pm 60 \%$. In the elastic region the line widths are not stress dependent within the limits of error which proofs that stress inhomogenities are negligible. As for $E / / P / /[001]$ line, we observed at about 22.8 $\mathrm{cm}^{-1}$ for $P>1 \mathrm{~kg} / \mathrm{mm}^{2}$ a possibly stress independent weak and sharp line emerging from the shifting line. It might be correlated to plastic deformations of $\mathrm{OH}^{-}$-impurities and will be investigated in further experiments.

We acknowledge experimental support by the Deutsche Forschungsgemeinschaft and helpful discussions with Dr. R. Weber. 


\section{References}

1. I. G. Nolt and A.J. Sievers, Phys. Rev. Letters 16 (1966) 1103; Bulletin 12 (1967) 79.

2. R. Weber and P. Nette, Phys. Letters 20 (1966) 493.

3. L. Genzel and R. Weber, Z. Angew. Physik 10 (1958) 195 ;

L. Genzel, J.Mol. Spectroscopy 4 (1960) 241.
4. G. F. Koster et al., Prop. of the thirty-two point groups (Cambridge, Mass., 1963).

5. W. Gebhardt and K. Maier, Phys. Stat.Sol. 8 (1965) 303.

6. G. Benedek and G. F. Nardelli, Phys. Rev. 167 (1968) 837.

7. J.T.Lewis, A.Lehoczky and C.V.Briscoe, Phys. Rev. 161 (1967) 877.

\title{
POTASSIUM-ELECTRON ELASTIC SPIN - EXCHANGE：COMPARISON OF EXPERIMENT WITH A CLOSE-COUPLING CALCULATION*
}

\author{
R. E. COLLINS, B. BEDERSON, M. GOLDSTEIN and K. RUBIN ** \\ New York University, University Heights, New York, New York 10453, USA
}

Received 19 June 1968

\begin{abstract}
Measurements are reported on the ratio of the differential exchange cross section to the full differential cross section for the elastic scattering of low energy electrons by potassium, for angles between $15^{\circ}$ and $180^{\circ}$ and energies between 0.5 and $1.2 \mathrm{eV}$.
\end{abstract}

Preliminary measurements at $0.5 \mathrm{eV}$ of the ratio of the differential spin-exchange cross section $\sigma_{\mathrm{ex}}(\theta)$ to the full differential cross section $\sigma(\theta)$ for potassium-electron elastic scattering have recently been reported [1]. This letter presents more complete experimental results at a number of electron energies, with considerably improved statistics and with reduced overall experimental error. The results are compared to the close-coupling approximation calculation of Karule [2] $\$$.

The experimental method has been described elsewhere [1,3]. Briefly, a spin- and velocityselected atom beam is cross-fired by an electron beam. The scattered atom beam profile is measured, with and without spin-state analysis, to obtain the quantity $R(\psi)$, the ratio of spin-flipped

\# We are indebted to Dr. Karule for sending us numerical values of singlet and triplet phase shifts for several electron energies. Values at other energies were determined from the partial cross sections tabulated by Karule in the above reference, using the relations

$$
\begin{aligned}
& \sigma_{l}^{+}=\frac{\pi}{k^{2}}(2 l+1) \sin ^{2} \eta_{l}^{+} \\
& \sigma_{\bar{l}}=\frac{3 \pi}{4 k^{2}}(2 l+1) \sin ^{2} \eta_{\bar{l}} .
\end{aligned}
$$

The ambiguity in modulo $\left(\pi-\eta_{l}\right)$ from these equations was resolved by comparison with the graphs of phase shifts versus energy presented by Dr. Karule. to full scattering atom beam currents at the atom scattering angle, $\psi$. For elastic scattering, a simple transformation into the electron polar scattering angle $\theta$ yields the quantity

$$
R(\theta) \equiv \frac{1}{2} \sigma_{\mathrm{ex}}(\theta) / \sigma(\theta) .
$$

Equivalently,

$$
R(\theta)=\frac{1}{2}|S-T|^{2} /\left(3|T|^{2}+|S|^{2}\right),
$$

where $S$ and $T$ are the singlet and triplet scattering amplitudes.

Experimental values of $\sigma_{\mathrm{ex}}(\theta) / \sigma(\theta)$ are presented in fig. 1 , for electron energies of 0.5 , $0.75,1.0$ and $1.2 \mathrm{eV}$. The electron energy spread in this work (f.w.h.m.) is $\sim 0.25 \mathrm{eV}$. The curves exhibit the same general behavior at all energies. The ratio is small at small angles, and increases rapidly in the vicinity of $\theta \approx 90^{\circ}$, to level off at a high value at large angles. This large-angle value increases with energy. The $1.2 \mathrm{eV}$ data exhibits a small maximum at $\theta \approx 90^{\circ}$. This effect is believed to be caused by a small amount of inelas -

\footnotetext{
* Supported by Army Research Office, Durham. N.C., Advanced Research Projects Agency through ONR, Washington, D.C., and the National Science Foundation.

** Permanent address: Physics Department, City University of New York, 138th Street and Convent Avenue, N.Y., N.Y.
} 